Comment. Math. Helv. 79 (2004) 798-825 0010-2571/04/040798-28

DOI $10.1007 / \mathrm{s} 00014-004-0805-1$
(C) 2004 Birkhäuser Verlag, Basel

Commentarii Mathematici Helvetici

\title{
A balancing condition for weak limits of families of minimal surfaces
}

Martin Traizet

\begin{abstract}
We prove a balancing condition for weak limits of families of embedded minimal surfaces of finite total curvature. We use it to prove compactness theorems for certain families of minimal surfaces.
\end{abstract}

Mathematics Subject Classification (2000). Primary 53A10.

Keywords. Minimal surface, compactness, forces.

The study of the moduli space of embedded complete minimal surfaces in $\mathbb{R}^{3}$ (up to homotheties) with finite total curvature and fixed topology is one of the fundamental open problems in the classical theory of minimal surfaces.

Let $\mathcal{M}(G, N)$ be the space of complete, properly embedded minimal surfaces in $\mathbb{R}^{3}$ with finite total curvature, genus $G$ and $N$ horizontal ends, modulo homotheties. Concerning the genus zero case, it is known that $\mathcal{M}(0,1)$ is the plane, $\mathcal{M}(0,2)$ is the catenoid, and $\mathcal{M}(0, N)$ is empty if $N \geq 3$ (see [4]). In higher genus, it is known that $\mathcal{M}(G, 2)$ is empty if $G \geq 1$ (see [10]), and $\mathcal{M}(1,3)$ is the Costa Hoffman Meeks family of genus one (see [1]). These are the only cases where $\mathcal{M}(G, N)$ is completely understood.

The following two conjectures have been proposed in [2], Section 5.2:

Conjecture 1 (the Hoffman Meeks conjecture). If $N \geq G+3, \mathcal{M}(G, N)$ is empty.

Conjecture 2. If $G \geq 1, \mathcal{M}(G, 3)$ is the set of Costa Hoffman Meeks surfaces of genus $G$.

In view of the recent proof of the uniqueness of the Riemann minimal examples by Meeks, Perez and Ros [5], a possible strategy to prove these conjectures would be to prove that if a counterexample exists, then one can deform it until it degenerates. One would obtain a contradiction by proving that the set of counterexamples is compact - actually compactness is also useful to prove that one can deform. 
The subject of this paper is to prove compactness theorems for families of minimal surfaces, under some geometric assumptions.

Using ideas of H. Choi, R. Shoen and B. White, Antonio Ros [8] has proven that the moduli space $\mathcal{M}(G, N)$ is weakly compact in the following sense: if $\left(M_{n}\right)_{n}$ is a sequence of embedded minimal surfaces with fixed genus $G$ and number of ends $N$, then up to passing to a subsequence, $\left(M_{n}\right)_{n}$ converges to a finite set of embedded minimal surfaces $\left\{M_{1, \infty}, \cdots, M_{m, \infty}\right\}$. Roughly speaking, this convergence means that when $n \rightarrow \infty$, the curvature concentrates in $m$ disjoint small balls $B_{1, n}, \cdots, B_{m, n}$, and after suitable blow-up, each sequence $M_{n} \cap B_{i, n}$ converges to $M_{i, \infty}$.

In this paper, we prove that the weak limit $\left\{M_{1, \infty}, \cdots, M_{m, \infty}\right\}$ satisfies a set of algebraic equations which we call the balancing condition: Theorem 4 . These equations relate the logarithmic growths of the ends and the "positions" of the limit surfaces $M_{1, \infty}, \cdots, M_{m, \infty}$. By the position of $M_{i, \infty}$, we mean the limit (up to some scaling) of the center of $B_{i, n}$. From the balancing condition, we deduce a single quadratic equation satisfied by the logarithic growths of the ends: Theorem 7 .

We then use these tools to prove compactness theorems. What we need to prove is that the sequence $\left(M_{n}\right)_{n}$ converges to a single limit $M_{1, \infty}$. Typically, we prove that if $m \geq 2$, then the quadratic equation has a sign, so it cannot be zero.

Concerning the first conjecture, we obtain the following result:

Theorem 1. Consider some $G \geq 1$ and assume that $\mathcal{M}\left(G^{\prime}, N^{\prime}\right)$ is empty for all $G^{\prime}<G$ and all $N^{\prime} \geq G^{\prime}+3$. Then for all $N \geq G+3, \mathcal{M}(G, N)$ is compact.

In other words, if the conjecture is known to be true for all $G^{\prime}<G$, then the set of counterexamples in the genus $G$ case is compact. This might be useful in an inductive proof of the conjecture.

Since the conjecture is known to be true for $G=0$, we obtain

Corollary 1. If $N \geq 4$, then $\mathcal{M}(1, N)$ is compact.

This was obtained by A. Ros in [8] when $N \geq 5$ using a quite different argument.

Regarding the second conjecture, we prove the following result. Let $M$ be an embedded minimal surface with $N$ ends. It is well known that the logarithmic growths of the ends satisfy $c_{1} \leq c_{2} \leq \cdots \leq c_{N}$.

Definition 1. We say an embedded minimal surface with $N$ ends has separated ends if $c_{1}<c_{2}<\cdots<c_{N}$.

Given $\varepsilon>0$, we say that the surface has $\varepsilon$-separated ends if $c_{k+1}-c_{k} \geq$ $\varepsilon\left(c_{N}-c_{1}\right)$ for all $1 \leq k \leq N-1$. Note that this condition if invariant by scaling.

We say that a sequence $\left(M_{n}\right)_{n}$ has uniformly separated ends if there exists $\varepsilon>0$ such that all $M_{n}$ have $\varepsilon$-separated ends. 
Let $\mathcal{C}(G)$ be the set of Costa Hoffman Meeks surfaces of genus $G$.

Theorem 2. Assume that $G \neq 2$. Let $\left(M_{n}\right)_{n}$ be a sequence of surfaces in $\mathcal{M}(G, 3) \backslash$ $\mathcal{C}(G)$ with uniformly separated ends. Then there exists a subsequence which converges to a single surface in $\mathcal{M}(G, 3)$.

We cannot guarantee that the limit is in $\mathcal{M}(G, 3) \backslash \mathcal{C}(G)$ because we do not know that $\mathcal{C}(G)$ is open.

Some nasty things happen in the genus 2 case, which suggests that surprisingly enough, the conjecture might be harder in this case.

The paper is organised as follows. We state our main result, the balancing condition, in Section 1. We prove it in Section 2. We give applications and prove Theorems 1 and 2 in Section 3.

\section{Main result}

\subsection{Weak compactness}

We first recall the weak compactness theorem of A. Ros:

Theorem 3. [8] Let $\left(M_{n}\right)_{n}$ be a sequence of non-flat embedded complete minimal surfaces in $\mathbb{R}^{3}$ with finite total curvature, horizontal ends and fixed topology: genus $G$ and $N$ ends. Then there exists a subsequence, denoted again by $\left(M_{n}\right)_{n}$, an integer $m>0$ and non-flat embedded complete minimal surfaces $M_{1, \infty}, \cdots, M_{m, \infty}$ with finite total curvature and horizontal ends, such that $\left(M_{n}\right)_{n}$ converges to $\left\{M_{1, \infty}, \cdots, M_{m, \infty}\right\}$. This convergence means the following:

1) $C\left(M_{n}\right)=C\left(M_{1, \infty}\right)+\cdots+C\left(M_{m, \infty}\right)$, where $C(M)$ means the total curvature of $M$.

2) There exists homotheties $\varphi_{i, n}$ such that $\varphi_{i, n}\left(M_{n}\right)$ converges smoothly to $M_{i, \infty}$ uniformly on compact subsets of $\mathbb{R}^{3}$.

3) Given $R, n$ large enough, let $B_{i, n}$ be the Euclidean ball $\varphi_{i, n}^{-1}(B(0, R))$. Then $B_{1, n}, \cdots, B_{m, n}$ are disjoint, and $M_{n}$ decomposes as

$$
M_{n}=M_{1, n} \cup \cdots \cup M_{m, n} \cup \Omega_{1, n} \cup \cdots \cup \Omega_{N, n}
$$

where $M_{i, n}=M_{n} \cap B_{i, n}$ and $\Omega_{k, n}$ is a graph over the exterior of some convex disks in the horizontal plane $x_{3}=0$, containing exactly one end of $M_{n}$.

We introduce some more terminology. We call $\Omega_{k, n}$ the unbounded domain at level $k$, and we say that the point at infinity in $\Omega_{k, n}$ is the end at level $k$ of $M_{n}$. Here the word "level" has a combinatorial meaning. To each end of $M_{i, \infty}$ corresponds one curve in $\partial M_{i, n}$ which lies in one domain $\Omega_{k, n}$. We say that the end under consideration is the end at level $k$ of $M_{i, \infty}$. 


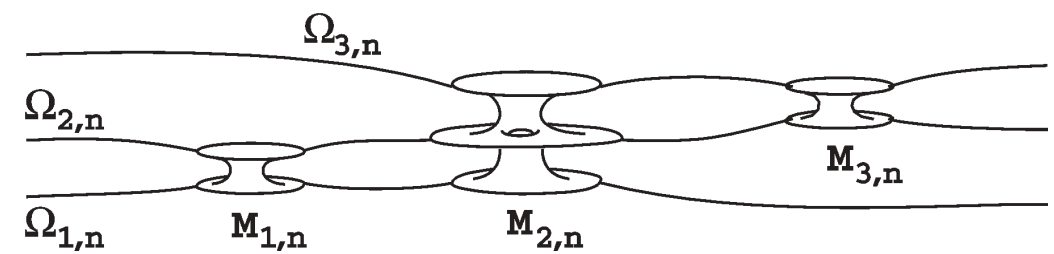

FIG. 1. A family of minimal surfaces $M_{n}$ whose weak limit is a Costa surface and two catenoids, one with ends at levels 1 and 2, the other with ends at levels 2 and 3.

\subsection{Uniform speed}

Definition 2. Let $\mu_{i, n}$ be the ratio of the homothety $\varphi_{i, n}$. We say the sequence $\left(M_{n}\right)_{n}$ has uniform speed if the $\mu_{i, n}$ are comparable, namely there exists a constant $C$ such that $\mu_{i, n} \leq C \mu_{j, n}$ for all $n, i, j$.

In Section 3.2 we will see a nice criterion for uniform speed, namely, if a sequence $\left(M_{n}\right)_{n}$ has uniformly separated ends, then it has uniform speed.

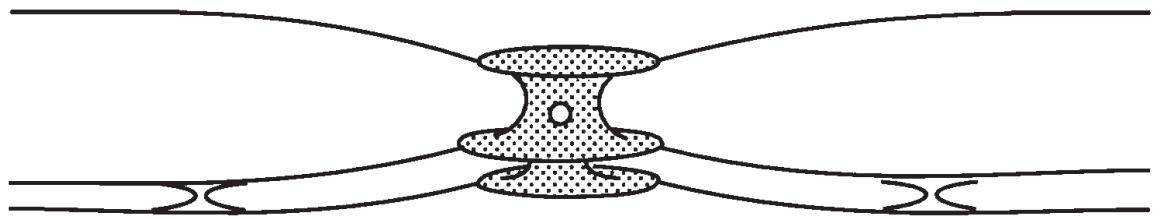

FIG. 2. A family of minimal surfaces $M_{n}$ with non-uniform speed. The two catenoids collapse faster than the 3-ended surface.

If the sequence has uniform speed, we may, by suitable scaling of $M_{n}$ and $M_{1, \infty}, \cdots, M_{m, \infty}$, assume that all $\mu_{i, n}$ are equal to 1 . (Indeed, by scaling of $M_{n}$ we may assume that $\mu_{1, n}=1$. Then passing to a subsequence, each $\mu_{i, n}$ has a limit $\ell_{i} \in(0, \infty)$. We replace $\varphi_{i, n}$ by $\varphi_{i, n} / \mu_{i, n}$ and $M_{i, \infty}$ by $M_{i, \infty} / \ell_{i}$.)

In case the sequence $\left(M_{n}\right)_{n}$ does not have uniform speed, we may still, by suitable scaling, assume that all $\mu_{i, n}$ are equal to 1 . However, some of the surfaces $M_{i, \infty}$ need to be scaled by zero, so they must be seen as multi-sheeted horizontal planes with a singular point. (Details: passing to a subsequence, we may assume that $\min \left\{\mu_{1, n}, \cdots, \mu_{m, n}\right\}=\mu_{i_{0}, n}$ with $i_{0}$ independent of $n$. By scaling of $M_{n}$ we may assume that $\mu_{i_{0}}=1$. Then passing to a subsequence, each $\mu_{i, n}$ has a limit $\ell_{i} \in[1, \infty]$. We replace as above $\varphi_{i, n}$ by $\varphi_{i, n} / \mu_{i, n}$ and $M_{i, \infty}$ by $M_{i, \infty} / \ell_{i}$. In case $\ell_{i}=\infty, M_{i, \infty}$ must be seen as a multi-sheeted horizontal plane.)

Let me summarise: $M_{n}$ and $M_{1, \infty}, \cdots, M_{m, \infty}$ have been scaled so that all $\varphi_{i, n}$ have ratio 1 so are in fact translations. Each limit $M_{i, \infty}$ is either a non-flat finite total curvature minimal surface, or a flat multi-sheeted horizontal plane. One 
basic problem is that our balancing condition will not "see" flat limits. Note that at least one limit surface is non-flat.

\subsection{The balancing condition}

Let $Q_{i, k}$ be the logarithmic growth of the end at level $k$ of $M_{i, \infty}$. Note that $Q_{i, k}=0$ if $M_{i, \infty}$ is flat. We also write $Q_{i, k}=0$ if $M_{i, \infty}$ has no end at level $k$. We shall interpret $Q_{i, k}$ as electrostatic charges. We define

$$
Q_{k}=\sum_{i=1}^{m} Q_{i, k}, \quad 1 \leq k \leq N .
$$

Then $Q_{k}$ is the limit of the logarithmic growth of the end at level $k$ of $M_{n}$ when $n \rightarrow \infty$.

Let $p_{i, n}$ be the projection of the center of the ball $B_{i, n}$ on the horizontal plane $x_{3}=0$. We see $p_{i, n}$ as a point in the complex plane. In general $p_{i, n} \rightarrow \infty$, so scaling is needed to see interesting limits. We may find homotheties $\psi_{n}$ such that up to a subsequence, each sequence $\psi_{n}\left(p_{i, n}\right)$ has a finite limit, which we call $p_{i}$, and moreover at least two points $p_{i}$ are distinct. We call $\left\{p_{1}, \cdots, p_{m}\right\}$ the limit configuration. We think of $p_{i}$ as the position of $M_{i, \infty}$, although we cannot see both $p_{i}$ and $M_{i, \infty}$ at the same scale.

Definition 3. We say the configuration is non-singular if the points $p_{1}, \cdots, p_{m}$ are distinct.

In this case we define forces by

$$
F_{i}=\sum_{k=1}^{N} \sum_{j \neq i} \frac{Q_{i, k} Q_{j, k}}{\overline{p_{i}}-\overline{p_{j}}} .
$$

Theorem 4 (non-singular case). In the above setup, if the limit configuration $\left\{p_{1}, \cdots, p_{m}\right\}$ is non-singular, then

1) the configuration is balanced: $\forall i, F_{i}=0$.

2) There exists numbers $H_{1}, \cdots, H_{N-1}$ such that the following holds: for any $i$, if $M_{i, \infty}$ has ends at levels $k$ and $k+1$, then

$$
Q_{i, k+1}-Q_{i, k}=H_{k} \text {. }
$$

The meaning of condition 2 is of course that if $i$ and $j$ are two such indices, then

$$
Q_{i, k+1}-Q_{i, k}=Q_{j, k+1}-Q_{j, k}
$$

We call 1 the balancing condition, or the force equation. We call 2 the charge equation. These are purely algebraic equations. They are invariant by scaling of the charges $Q_{i, k}$ and scaling/rotation/translation of the points $p_{1}, \cdots, p_{m}$. 


\subsection{The singular case}

Assume now that the configuration $\left\{p_{1}, \cdots, p_{m}\right\}$ is singular. Define an equivalence relation on $\{1, \cdots, m\}$ by $i \sim j$ if $p_{i}=p_{j}$. Given an equivalence class $\alpha$, write $p_{\alpha}$ for the common value of all $p_{i}, i \in \alpha$. Define charges and forces by

$$
\begin{gathered}
Q_{\alpha, k}=\sum_{i \in \alpha} Q_{i, k} \\
F_{\alpha}=\sum_{k=1}^{N} \sum_{\beta \neq \alpha} \frac{Q_{\alpha, k} Q_{\beta, k}}{\overline{p_{\alpha}}-\overline{p_{\beta}}}
\end{gathered}
$$

where the last sum is taken on all equivalence classes $\beta$ (possibly with only one point) different from $\alpha$. As we shall see, the configuration is balanced in the sense that $F_{\alpha}=0$ for all classes $\alpha$.

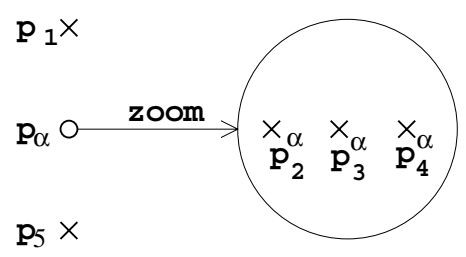

FIG. 3. A singular configuration, with $\alpha=\{2,3,4\}$. The configuration $\left\{p_{1}, p_{\alpha}, p_{5}\right\}$ is balanced. The sub-configuration $\left\{p_{2}^{\alpha}, p_{3}^{\alpha}, p_{4}^{\alpha}\right\}$ is balanced.

We may say more by zooming in. Let $\alpha$ be a class with at least two elements. We may find homotheties, denoted $\psi_{n}^{\alpha}$, such that the sequence $\psi_{n}^{\alpha}\left(p_{i, n}\right)$ has a finite limit for each $i \in \alpha$, denoted $p_{i}^{\alpha}$, and at least two of these points are different. (Clearly, $\psi_{n}^{\alpha}\left(p_{j, n}\right) \rightarrow \infty$ when $j \notin \alpha$.) We call $\left\{p_{i}^{\alpha}, i \in \alpha\right\}$ a sub-configuration. If the sub-configuration $p_{i}^{\alpha}$ is non-singular (which means, of course, that all points $p_{i}^{\alpha}, i \in \alpha$, are distinct), we may define forces by

$$
F_{i}^{\alpha}=\sum_{k=1}^{N} \sum_{\substack{j \in \alpha \\ j \neq i}} \frac{Q_{i, k} Q_{j, k}}{\overline{p_{i}^{\alpha}}-\overline{p_{j}^{\alpha}}} .
$$

Theorem 5 (singular case, depth 2). In the above setup, assume that all subconfigurations are non-singular, then:

1a) each sub-configuration if balanced, in the sense that $F_{i}^{\alpha}=0$ for all $\alpha$ and all $i \in \alpha$.

1b) The configuration is balanced, in the sense that $F_{\alpha}=0$ for all classes $\alpha$.

2a) For each $\alpha$, there exists numbers $H_{k}^{\alpha}$ such that the following holds: for each $i \in \alpha$, if $M_{i, \infty}$ has ends at levels $k$ and $k+1$, then

$$
Q_{i, k+1}-Q_{i, k}=H_{k}^{\alpha} \text {. }
$$


2b) For each $\alpha$ and each $i \in \alpha$ such that $M_{i, \infty}$ has ends at levels $k$ and $k+1$, there exists $\rho \in[0,1]$ such that

$$
\rho\left(Q_{i, k+1}-Q_{i, k}\right)+(1-\rho)\left(Q_{\alpha, k+1}-Q_{\alpha, k}\right)=H_{k} .
$$

where $H_{k}$ is as in Theorem 4 .

The statements 1a) and 2a) say that each sub-configuration satisfies the conclusion of Theorem 4. The statement $1 \mathrm{~b}$ ) says that as far as forces are concerned, we may see all surfaces $M_{i, \infty}, i \in \alpha$ as one single surface with ends of logarithmic growths $Q_{\alpha, k}$.

To get some grasp on point 2b), observe that if $\rho=1$ then we get $H_{k}=$ $Q_{i, k+1}-Q_{i, k}$ as if the configuration were non-singular, while if $\rho=0$, we get $H_{k}=Q_{\alpha, k+1}-Q_{\alpha, k}$ as if all surfaces $M_{i, \infty}, i \in \alpha$, were one single surface with logarithmic growths $Q_{\alpha, k}$. It is very reasonable that both cases might happen, so one cannot hope for a better result.

We will use $2 \mathrm{~b}$ ) as follows: if $p_{j}$ is a non-singular point of the configuration and $M_{j, \infty}$ has ends at levels $k$ and $k+1$, then $Q_{j, k+1}-Q_{j, k}$ is in the interval bounded by $Q_{i, k+1}-Q_{i, k}$ and $Q_{\alpha, k+1}-Q_{\alpha, k}$. In other words, in the singular case, the charge equation must be replaced by some inequalities (see Section 3.3.2. for an example).

There are no known examples of families of minimal surfaces which give in the limit a singular configuration (figure 4 is for illustration only). However, in [11], examples are constructed for which the configuration is arbitrary close to be singular. This strongly suggests that singular configurations are possible.

Of course, there might be singular sub-configurations. In this case, by zooming in again, we see balanced sub-sub-configurations, and so on. By successive zoomings like this we obtain what we call a nested configuration. We have a similar theorem in this case. This will be explained in Section 2.5.

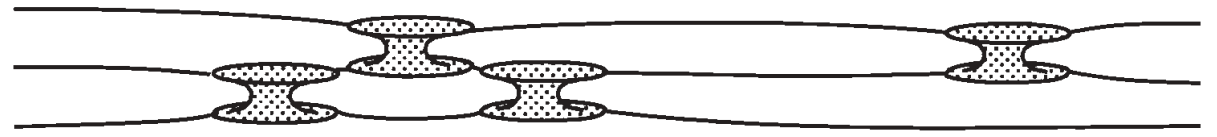

FIG. 4. A family of minimal surfaces which gives a singular configuration. The three catenoids on the left converge to the same point.

\subsection{Related work}

This paper stems from a failed attempt by the author [11] to construct counterexamples to the conjectures 1 and 2. In [11], I proved the converse to Theorem 4 in 
the case where all surfaces $M_{i, \infty}$ are catenoids under an additional non-degeneracy hypothesis. Namely if we start from a balanced, non-degenerate, non-singular configuration of catenoids, we can construct a family of minimal surfaces.

I was then able to classify all configurations in the 3-ended case, and unfortunately found only the ones which yield the Costa Hoffman Meeks family (except in the genus 2 case). Still, it is quite interesting to see how the balancing condition forces the configuration to be very symmetric. To me this was good support to the conjecture.

Fortunately, [11] was more successful in answering other questions, in particular I proved the existence of embedded minimal surfaces with no symmetries.

To prove compactness theorems, it is necessary to allow limit surfaces other than catenoids, and one has to consider the case where the configuration is singular, which makes things much more complicated.

\section{Proof}

Our plan is to identify the domain $\Omega_{k, n}$ with a domain in the complex plane, and compute the limit of its Weierstrass data when $n \rightarrow \infty$. We obtain the force equation $F_{i}=0$ by computing the limit of the horizontal flux along certain cycles. We obtain the charge equation by estimating the height between the domains $\Omega_{k, n}$ and $\Omega_{k+1, n}$.

\subsection{Preliminaries}

We introduce some notations: $g_{n}$ and $d h_{n}$ are the Gauss map and height differential of $M_{n}, \lambda_{n}$ is the ratio of $\psi_{n}, I_{k}$ is the set of indices $i$ such that $M_{i, \infty}$ has an end at level $k$. For $i \in I_{k}, \gamma_{i, k, n}$ is the curve at level $k$ in the boundary of $M_{i, n}$, oriented as a boundary, so $-\gamma_{i, k, n}$ is oriented as a boundary of $\Omega_{k, n}$.

We now fix some level $k, 1 \leq k \leq N$ and consider the domain $\Omega_{k, n}$. As $\Omega_{k, n}$ is a graph, we may (changing orientation if necessary) assume that the normal points up, so that $\left|g_{n}\right|>1$ in $\Omega_{k, n}$. Let $\zeta_{n}: \Omega_{k, n} \rightarrow \mathbb{C}$ be the composition of the projection on the horizontal plane followed with the homothety $\psi_{n}$. Clearly $\zeta_{n}\left(\Omega_{k, n}\right)$ converges to $\mathbb{C} \backslash\left\{p_{i}, i \in I_{k}\right\}$.

We need to introduce a global conformal coordinate $z_{n}$ on $\Omega_{k, n}$. We shall do this so that $z_{n}$ is as close as possible to $\zeta_{n}$ (which preserves orientation but is not conformal). By Koebe's Theorem on the uniformisation of multiply connected domains, (see [9] and the reference therein), $\Omega_{k, n}$ is conformally equivalent to a canonical circle domain, namely, there exists a conformal representation $z_{n}$ of $\Omega_{k, n}$ onto a domain of the form $\mathbb{C}$ minus round disks $D\left(a_{i, n}, r_{i, n}\right), i \in I_{k}$. Moreover, $z_{n}$ is unique up to a Moebius transform. We normalise $z_{n}$ by asking that $z_{n}=\zeta_{n}$ at three points, which we choose to be $\infty$ and two other fixed points far from the 
points $p_{i}$. We need the following estimates:

Proposition 1. When $n \rightarrow \infty$, we have (up to subsequences) $z_{n} \sim \zeta_{n}$ in the sense that $z_{n}\left(\zeta_{n}^{-1}(z)\right) \rightarrow z$ uniformly on compact subsets of $\mathbb{C} \backslash\left\{p_{i}, i \in I_{k}\right\}$. In particular, we have $\lim a_{i, n}=p_{i}$ and $\lim r_{i, n}=0$. Moreover, we have

$$
\lim _{R \rightarrow \infty} \lim _{n \rightarrow \infty} \frac{\log r_{i, n}}{\log \lambda_{n}}=1
$$

where $R$ is the radius of $B_{i, n}$ in Theorem 3.

Proof. Note that $\Omega_{k, n}, \gamma_{i, k, n}$ and $z_{n}$ all depend on $R$. We will most often fix the value of $R$, but we sometime need to let $R \rightarrow \infty$. When this happens, we will always let first $n \rightarrow \infty$ and then $R \rightarrow \infty$.

It will be convenient to replace the ball $B(0, R)$ in the statement of Theorem 3 by a vertical cylindrical box of radius $R$ and height $2 R$ (in other words, we use the norm $\|x\|=\max \left(\sqrt{x_{1}^{2}+x_{2}^{2}},\left|x_{3}\right|\right)$ to define balls in $\left.\mathbb{R}^{3}\right)$. This clearly does not change anything to the statement of the theorem. Thus the projection of $\gamma_{i, k, n}$ on the horizontal plane is the circle $C\left(p_{i, n}, R\right)$, and $\zeta_{n}\left(\gamma_{i, k, n}\right)$ is a circle of radius $\lambda_{n} R$.

As $1 / g_{n}$ is holomorphic in $\Omega_{k, n}$, its maximum is on the boundary. From the convergence of $M_{i, n}$ to $M_{i, \infty}$, we obtain

$$
\lim _{R \rightarrow \infty} \lim _{n \rightarrow \infty} \inf _{\Omega_{k, n}}\left|g_{n}\right|=\infty
$$

Hence $\zeta_{n}$ is $\kappa$-quasiconformal on $\Omega_{k, n}$, with $\kappa \rightarrow 1$ when $n \rightarrow \infty$ and $R \rightarrow \infty$.

Now fix some $R$ and let $K$ be a compact subset of $\mathbb{C} \backslash\left\{p_{i}, i \in I_{k}\right\}$. Then

$$
\lim _{n \rightarrow \infty} \inf _{\zeta_{n}^{-1}(K)}\left|g_{n}\right|=\infty
$$

so $\zeta_{n}$ restricted to $K$ is $\kappa$-quasiconformal with $\kappa \rightarrow 1$ when $n \rightarrow \infty$. Since $z_{n} \circ \zeta_{n}^{-1}$ : $K \rightarrow \mathbb{C}$ is $\kappa$-quasiconformal and fixes three points, we may extract a converging subsequence by some standard normal family argument ([3], Proposition 5.1 page 73). Using an exhausting sequence of compact sets, $z_{n} \circ \zeta_{n}^{-1}$ converges on compact subsets of $\mathbb{C} \backslash\left\{p_{i}, i \in I_{k}\right\}$ to a holomorphic function $f: \mathbb{C} \backslash\left\{p_{i}, i \in I_{k}\right\} \rightarrow \mathbb{C} . f$ clearly extends to a holomorphic bijection of $\mathbb{C} \cup\{\infty\}$. Since $f$ fixes three points, it has to be the identity, which proves the first statements.

To prove the last statement, consider the annular domain in $\Omega_{k, n}$ defined by $r_{i, n}<\left|z_{n}-a_{i, n}\right|<\varepsilon$ for some fixed small $\varepsilon>0$. The image of this domain by $\zeta_{n}$ is bounded by a circle of radius $\lambda_{n} R$ and a curve which converges to the circle $C\left(p_{i}, \varepsilon\right)$ when $n \rightarrow \infty$. Since $\zeta_{n}$ is $\kappa$-quasiconformal, the moduli of these domains differ by a factor at most $\kappa$. The moduli are by definition $\log \varepsilon-\log r_{i, n}$ and $\log \varepsilon-\log \left(\lambda_{n} R\right)$. Since $\kappa \rightarrow 1$ when $R \rightarrow \infty$, the last statement follows. 


\subsection{Limit of the Weierstrass data}

We identify $\Omega_{k, n}$ with a domain in the complex plane by mean of the conformal coordinate $z_{n}$, that is, we write $z_{n}=z$.

Proposition 2. Up to a subsequence, we have on $\Omega_{k, n}$

$$
\begin{gathered}
\lim _{n \rightarrow \infty} d h_{n}=\sum_{i \in I_{k}} \frac{Q_{i, k}}{z-p_{i}} d z \\
\lim _{n \rightarrow \infty} \lambda_{n} g_{n} d h_{n}=-2 d z
\end{gathered}
$$

The convergence is uniform on compact subsets of $\mathbb{C} \backslash\left\{p_{i}, i \in I_{k}\right\}$ and is also uniform with respect to $R$.

Proof. Let $d h_{i, \infty}$ be the height differential of $M_{i, \infty}$. Since $M_{i, \infty}$ has horizontal catenoid (or planar) type ends, $d h_{i, \infty}$ has at most a simple pole at each end, so there exists a constant $C$ such that

$$
\forall R, \quad \int_{\partial\left(M_{i, \infty} \cap B(0, R)\right)}\left|d h_{i, \infty}\right| \leq C .
$$

Up to the translation $\varphi_{i, n}, M_{n}$ converges to $M_{i, \infty}$ so we have for $n$ large enough

$$
\int_{\gamma_{i, k, n}}\left|d h_{n}\right| \leq C
$$

By definition of $Q_{i, k}$, we have

$$
\lim _{n \rightarrow \infty} \int_{\gamma_{i, k, n}} d h_{n}=2 \pi \mathrm{i} Q_{i, k}
$$

By elementary complex analysis, Lemma 1 below with $J=\emptyset, d h_{n}$ converges to a meromorphic differential with at most simple poles at $p_{i}, i \in I_{k}$. The residue at $p_{i}$ is $Q_{i, k}$ (provided the $p_{i}$ are distinct). This determines the limit, and proves the first statement of the proposition. When several $p_{i}$ are equal, the residues add up, and the statement still holds.

To prove the second statement, recall that $\zeta_{n} \sim z_{n}=z$ which gives

$$
d z \sim d \zeta_{n}=\lambda_{n} \frac{1}{2}\left(\overline{g_{n}^{-1} d h_{n}}-g_{n} d h_{n}\right)
$$

Since $g_{n} \rightarrow \infty$ on compact subsets of $\mathbb{C} \backslash\left\{p_{i}\right\}$, this gives the second statement of the proposition.

For future reference, we state a lemma in a setting more general than needed here.

Lemma 1. Consider a sequence of domains $\Omega_{n}=\mathbb{C} \backslash \bigcup_{i \in I \cup J} D\left(a_{i, n}, r_{i, n}\right)$, where $I$ and $J$ are finite sets, $\lim a_{i, n}=p_{i} \neq \infty$ when $i \in I, \lim a_{i, n}=\infty$ when $i \in J$, 
and $\lim r_{i, n}=0$. Let $f_{n}: \Omega_{n} \rightarrow \mathbb{C}$ be a sequence of holomorphic functions such that

$$
\int_{\partial \Omega_{n}}\left|f_{n}\right| \leq C
$$

and $f_{n}(z) d z$ has at most a simple pole at $\infty$. Then there exists a subsequence, still noted $f_{n}$, which converges on compact subsets of $\mathbb{C} \backslash\left\{p_{i}, i \in I\right\}$ to a meromorphic function $f$. Moreover $f$ has at most simple poles at each $p_{i}, i \in I$, and $f(z) d z$ has at most a simple pole at $\infty$.

Proof. Consider some $\varepsilon>0$ and let $U_{\varepsilon}$ be the set of $z$ such that $\forall i \in I,\left|z-p_{i}\right| \geq \varepsilon$. Then for $n$ large enough, any $z \in U_{\varepsilon}$ is at distance at least $\varepsilon / 2$ from $\partial \Omega_{n}$. By the residue theorem (note that the integrand has no pole at $\infty$ )

$$
\left|f_{n}(z)\right|=\left|\frac{1}{2 \pi i} \int_{\partial \Omega_{n}} \frac{f_{n}(w)}{w-z} d w\right| \leq \frac{C}{\pi \varepsilon}
$$

Hence we may extract a subsequence $f_{n}$ which converges on $U_{\varepsilon}$. By a diagonal process, we may extract a subsequence which converges on $U_{\varepsilon}$ for all $\varepsilon$. Let $f=\lim f_{n}$. The above estimate implies that for any $\varepsilon>0$,

$$
\int_{\left|z-p_{i}\right|=\varepsilon}|f(z) d z| \leq 2 C
$$

It easily follows, using a Laurent series, that $f$ has at most a simple pole at $p_{i}$.

\subsection{The balancing condition}

Let $\gamma$ be a closed curve on a minimal surface. The flux of $\gamma$ is the vector flux $(\gamma)=$ $\int_{\gamma} \nu d s$ where $\nu$ is the conormal to $\gamma$. Physically, the flux is the force exerted by the surface on $\gamma$, so may be observed in soap film experiments, see figure 5 .

Let $F(\gamma)$ be the horizontal part of flux $(\gamma)$, seen as a complex number. In term of Weierstrass Representation,

$$
F(\gamma)=\frac{i}{2}\left(\int_{\gamma} \overline{g^{-1} d h}+\int_{\gamma} g d h\right)
$$

Since $\gamma$ is a closed curve, we have

$$
\int_{\gamma} \overline{g^{-1} d h}=\int_{\gamma} g d h
$$

so

$$
F(\gamma)=i \int_{\gamma} \overline{g^{-1} d h}=i \int_{\gamma} g d h .
$$




\subsubsection{The non-singular case}

Proposition 3 (non-singular case). Assume that all points $p_{i}, i \in I_{k}$, are distinct. Then for $i \in I_{k}$,

$$
\lim _{n \rightarrow \infty} \frac{1}{\lambda_{n}} F\left(\gamma_{i, k, n}\right)=-2 \pi \sum_{j \neq i} \frac{Q_{i, k} Q_{j, k}}{\bar{p}_{i}-\bar{p}_{j}}
$$

Since the curves $\gamma_{i, k, n}$ bound the minimal surface $M_{i, n}$, we have

$$
\sum_{k} F\left(\gamma_{i, k, n}\right)=0
$$

where the sum is taken on all $k$ such that $M_{i, \infty}$ has an end at level $k$. This gives the balancing condition

$$
\forall i, \quad-2 \pi \sum_{k} \sum_{j \neq i} \frac{Q_{i, k} Q_{j, k}}{\bar{p}_{i}-\bar{p}_{j}}=0 .
$$

Remark 1. The proposition predicts that when $Q_{i, k}$ and $Q_{j, k}$ have the same sign, the force between them is attractive. This is in agreement with an interesting soap film experiment, see figure 5. We start with a soap film bounded by three wire circles in the same horizontal plane. The two small circles are hanging on threads. When we carefully lift up the small circles, they are attracted to each other. When we move one up and one down, they are repelled from each other. The first experiment was explained to me by A. Ros and is described in [6], Section 2.1. The second one was suggested by Proposition 3.

Note that if we interpret $Q_{i, k}$ and $Q_{j, k}$ as electrostatic charges, the force between them should be repulsive when they have the same sign. This is the reason why we defined the force $F_{i}$ with the opposite sign.
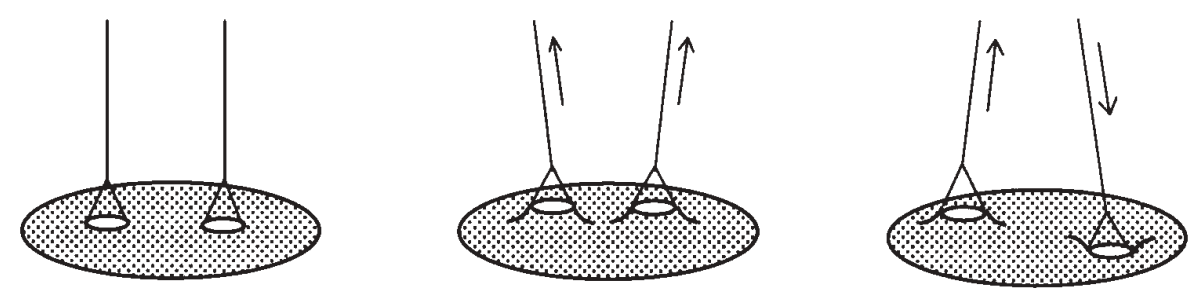

FIG. 5. A soap film experiment. 
Proof. Observe that if we change the orientation of $M_{n}$, then $\gamma_{i, k, n}, g_{n}$ and $d h_{n}$ are changed into $-\gamma_{i, k, n},-1 / \overline{g_{n}}$ and $\overline{d h_{n}}$, so $F\left(\gamma_{i, k, n}\right)$ is not changed. This was to be expected, of course, given the physical interpretation of the flux as a force. Hence, we may assume that the Gauss map points up in $\Omega_{k, n}$ and use the results of the preceding sections.

$$
\begin{aligned}
& \lim _{n \rightarrow \infty} \frac{1}{\lambda_{n}} \overline{F\left(\gamma_{i, k, n}\right)}=\lim _{n \rightarrow \infty}-\frac{\mathrm{i}}{\lambda_{n}} \int g_{n}^{-1} d h_{n}=\lim _{n \rightarrow \infty}-i \int\left(d h_{n}\right)^{2}\left(\lambda_{n} g_{n} d h_{n}\right)^{-1} \\
& =\frac{\mathrm{i}}{2} \int\left(\sum_{j} \frac{Q_{j, k}}{z-p_{j}}\right)^{2}=-\pi \operatorname{Res}_{p_{i}}\left(\sum_{j} \frac{Q_{j, k}}{z-p_{j}}\right)^{2} \\
& =-\pi \operatorname{Res}_{p_{i}}\left(\frac{Q_{i, k}^{2}}{\left(z-p_{i}\right)^{2}}+2 \sum_{j \neq i} \frac{Q_{i, k} Q_{j, k}}{\left(z-p_{i}\right)\left(z-p_{j}\right)}+\sum_{j, \ell \neq i} \frac{Q_{j, k} Q_{\ell, k}}{\left(z-p_{j}\right)\left(z-p_{\ell}\right)}\right) \\
& =-2 \pi \sum_{j \neq i} \frac{Q_{i, k} Q_{j, k}}{p_{i}-p_{j}} .
\end{aligned}
$$

In the above computation, we computed the integral on the circle $C\left(p_{i}, \varepsilon\right)$ which is homologous to $\gamma_{i, k, n}$ and where Proposition 2 applies ( $\varepsilon$ is a fixed small number).

\subsubsection{The singular case}

We now explain how to adapt the above argument in the case where the configuration is singular but each sub-configuration is non-singular, as in Theorem 5 . In this case, we have, with the notations of Section 1.4,

$$
\lim _{n \rightarrow \infty} d h_{n}=\sum_{\alpha} \frac{Q_{\alpha, k}}{z-p_{\alpha}} .
$$

Let $\gamma_{\alpha, k, n}$ be the circle $C\left(p_{\alpha}, \varepsilon\right)$ in $\Omega_{k, n}$. The above computation gives

$$
\lim _{n \rightarrow \infty} \frac{1}{\lambda_{n}} F\left(\gamma_{\alpha, k, n}\right)=-2 \pi \sum_{\beta \neq \alpha} \frac{Q_{\alpha, k} Q_{\beta, k}}{\bar{p}_{\alpha}-\bar{p}_{\beta}} .
$$

Summing on $k$, we obtain that the configuration is balanced. This proves point 1b) of Theorem 5. Note that we do not need that the sub-configurations are non-singular here.

To prove that the sub-configuration obtained by zooming on $p_{\alpha}$ is balanced, namely $F_{i}^{\alpha}=0$, we do the exact same thing, replacing the homothety $\psi_{n}$ by $\psi_{n}^{\alpha}$ when we define $\zeta_{n}$. The only difference is that some points go to $\infty$, so $J \neq \emptyset$ when we use Lemma 1. 


\subsection{Height estimate}

\subsubsection{The non-singular case}

We fix some point $A \in \mathbb{C}$ away from all points $p_{i}$, and let $A_{k, n}$ be the point $z_{n}=A$ in $\Omega_{k, n}$. Recall that $\lambda_{n} \rightarrow 0$.

Proposition 4. Let $i \in I_{k} \cap I_{k+1}$. Assume the configuration is non-singular. Then

$$
\lim _{n \rightarrow \infty} \frac{-1}{\log \lambda_{n}} \operatorname{Re} \int_{A_{k, n}}^{A_{k+1, n}} d h_{n}=Q_{i, k+1}-Q_{i, k} .
$$

Up to the $\log$ factor, the left side is the height between $A_{k, n}$ and $A_{k+1, n}$ so does not depend on the path of integration. This proves that $Q_{i, k+1}-Q_{i, k}$ is the same for all $i \in I_{k} \cap I_{k+1}$.

Proof. Let $P_{i, k, n}$ be the point $z_{n}=a_{i, n}+2 r_{i, n}$ in $\Omega_{k, n}$. From the convergence of $M_{i, n}$ to $M_{i, \infty}$ we have

$$
\operatorname{Re} \int_{P_{i, k, n}}^{P_{i, k+1, n}} d h_{n}=\mathcal{O}(\log R)
$$

where we compute the integral on a path which goes through $M_{i, n}$. Lemma 2 below gives

$$
\operatorname{Re} \int_{A_{k, n}}^{P_{i, k, n}} d h_{n}=\left(Q_{i, k}+\varepsilon(n)\right) \log r_{i, n}+\mathcal{O}(1)
$$

where as usual $\varepsilon(n)$ means a function which goes to 0 when $n \rightarrow \infty$, uniformly with respect to $R$. Using Proposition 1, we obtain

$$
\operatorname{Re} \int_{A_{k, n}}^{A_{k+1, n}} d h_{n}=\left(Q_{i, k}-Q_{i, k+1}+\varepsilon(n)\right)(1+\varepsilon(R)) \log \lambda_{n}+\mathcal{O}(R) .
$$

This gives

$$
\lim _{n \rightarrow \infty} \frac{-1}{\log \lambda_{n}} \operatorname{Re} \int_{A_{k, n}}^{A_{k+1, n}} d h_{n}=\left(Q_{i, k+1}-Q_{i, k}\right)(1+\varepsilon(R)) .
$$

The result follows by letting $R \rightarrow \infty$.

Lemma 2. With the hypotheses of Lemma 1, assume further that all $p_{i}, i \in I$ are distinct. Fix some small $\varepsilon>0$ and consider some $z$ in the annular region $2 r_{i, n} \leq\left|z-a_{i, n}\right| \leq \varepsilon$. (Here the 2 might be replaced by any number $>1$ ). Then when $n \rightarrow \infty$,

$$
\int_{z_{0}}^{z} f_{n}(z) d z=\left(\frac{1}{2 \pi i} \int_{\partial D_{i, n}} f_{n}(z) d z\right) \log \left|z-a_{i, n}\right|+\mathcal{O}(1)
$$

where $\mathcal{O}(1)$ means a function which is uniformly bounded with respect to $z$ and $n$. 
Proof. By Lemma 1, the integral of $f_{n}$ from $z_{0}$ to $a_{i, n}+\varepsilon$ is a bounded function. To estimate the integral from $a_{i, n}+\varepsilon$ to $z$, we write the Laurent series of $f_{n}$ in the annular region $r_{i, n}<\left|z-a_{i, n}\right|<2 \varepsilon$ :

$$
f_{n}(z)=\sum_{m \in \mathbb{Z}} c_{m}\left(z-a_{i, n}\right)^{m}
$$

where the coefficients $c_{m}$ depend on $n$ and are given by

$$
c_{m}=\frac{1}{2 \pi i} \int_{\left|z-a_{i, n}\right|=2 \varepsilon} \frac{f_{n}(z)}{\left(z-a_{i, n}\right)^{m+1}} d z=\frac{1}{2 \pi i} \int_{\left|z-a_{i, n}\right|=r_{i, n}} \frac{f_{n}(z)}{\left(z-a_{i, n}\right)^{m+1}} d z .
$$

From the proof of Lemma 1, we have

Now we compute

$$
\begin{aligned}
& \int_{\left|z-a_{i, n}\right|=2 \varepsilon}|f| \leq 2 C \quad \Longrightarrow \quad\left|c_{m}\right| \leq \frac{C^{\prime}}{(2 \varepsilon)^{m+1}}, \\
& \int_{\left|z-a_{i, n}\right|=r_{i, n}}|f| \leq C \quad \Longrightarrow \quad\left|c_{m}\right| \leq \frac{C^{\prime}}{\left(r_{i, n}\right)^{m+1}} .
\end{aligned}
$$

$$
\int_{a_{i, n}+\varepsilon}^{z} f_{n}(z) d z=c_{-1} \log \frac{z-a_{i, n}}{\varepsilon}+\sum_{m \neq-1} \frac{c_{m}}{m+1}\left(\left(z-a_{i, n}\right)^{m+1}-\varepsilon^{m+1}\right) .
$$

The first term is what we want. Using the first and second estimates of $c_{m}$, it is straightforward to see that the sum for $m \geq 0$ and $m \leq-2$, respectively, are bounded.

\subsubsection{The singular case}

We now consider the case where the configuration is singular and each subconfiguration is non-singular, as in Theorem 5. We define a conformal coordinate $z_{n}^{\alpha}$ on $\Omega_{k, n}$ using $\psi_{n}^{\alpha}$ instead of $\psi_{n}$ in the definition of $z_{n}$. Let $A_{k, n}^{\alpha}$ be the point $z_{n}^{\alpha}=A$ in $\Omega_{k, n}$. Then by Proposition 4 , we have when $n \rightarrow \infty$

$$
\operatorname{Re} \int_{A_{k, n}^{\alpha}}^{A_{k+1, n}^{\alpha}} d h_{n} \sim\left(Q_{i, k}-Q_{i, k+1}\right) \log \lambda_{n}^{\alpha}
$$

This proves point $2 \mathrm{a}$ ) of Theorem 5 .

To evaluate the integral from $A_{k, n}$ to $A_{k, n}^{\alpha}$, we go back to the coordinate $z_{n}$. When we identify $\Omega_{k, n}$ with a domain in the complex plane via $z_{n}$, we have on compact subsets of $\mathbb{C} \backslash\left\{p_{\alpha}\right\}$,

$$
\lim d h_{n}=\sum_{\alpha} \frac{Q_{\alpha, k}}{z-p_{\alpha}} d z
$$

Also for the point $z_{n}^{\alpha}=A$, we have $z_{n}-a_{i, n} \sim A \lambda_{n} / \lambda_{n}^{\alpha}$. The proof of Proposition 4 gives

$$
\operatorname{Re} \int_{A_{k, n}}^{A_{k, n}^{\alpha}} d h_{n} \sim Q_{\alpha, k} \log \frac{\lambda_{n} A}{\lambda_{n}^{\alpha}}
$$


This gives

$\frac{1}{\log \lambda_{n}} \operatorname{Re} \int_{A_{k, n}}^{A_{k+1, n}} d h_{n} \sim\left(Q_{i, k}-Q_{i, k+1}\right) \frac{\log \lambda_{n}^{\alpha}}{\log \lambda_{n}}+\left(Q_{\alpha, k}-Q_{\alpha, k+1}\right) \frac{\log \lambda_{n}-\log \lambda_{n}^{\alpha}}{\log \lambda_{n}}$.

Passing to a subsequence we may assume that (recall that $0<\lambda_{n}<\lambda_{n}^{\alpha}<1$ )

$$
\rho=\lim _{n \rightarrow \infty} \frac{\log \lambda_{n}^{\alpha}}{\log \lambda_{n}} \in[0,1]
$$

exists. This proves point $2 \mathrm{~b}$ ) of Theorem 5 .

\subsection{The case of nested configurations}

As was explained at the end of Section 1.4, in the case of singular configurations, we may have to make several successive zooms before we see non-singular subconfigurations. This is illustrated in figure 6 . We construct by this process a tree, whose leaves are labelled with the indices $1, \cdots, m$, and whose nodes are labelled by subsets of $\{1, \cdots, m\}$ : each node is labelled by the set of leaves that are below it. The root is labelled with $\{1, \cdots, m\}$ and corresponds to the full configuration.

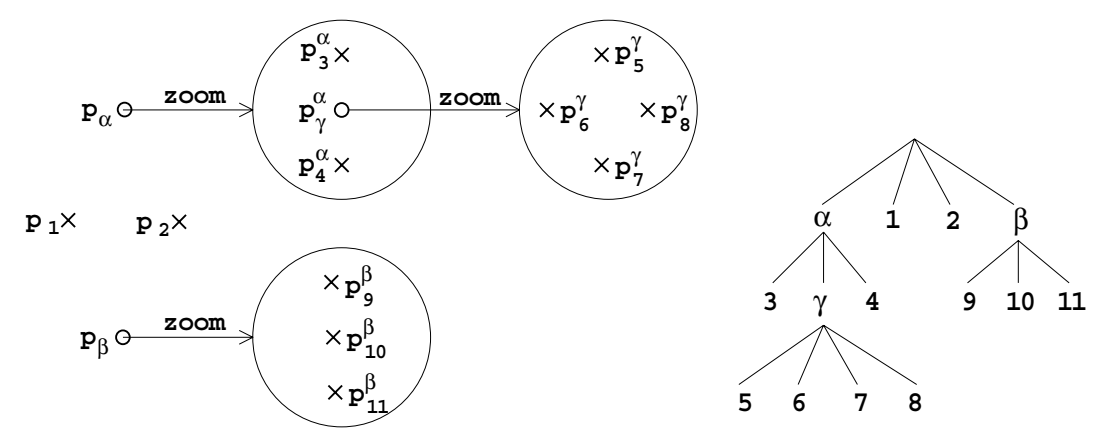

FIG. 6. A nested singular configuration of depth 3 and the corresponding tree, with $\alpha=\{3,4,5,6,7,8\}, \beta=\{9,10,11\}$ and $\gamma=\{5,6,7,8\}$.

The depth of a leaf is its distance to the root. The depth of a configuration is the maximum depth of its leaves. For example, a non-singular configuration has depth 1.

To each node $\alpha$ is associated a sequence of homotheties $\psi_{n}^{\alpha}$, such that for each son $\beta$ of $\alpha$ and each $i \in \beta, \lim \psi_{n}^{\alpha}\left(p_{i, n}\right)$ exists and only depend on $\beta$. We call it $p_{\beta}^{\alpha}$. Moreover, for each $\alpha$, at least two $p_{\beta}^{\alpha}$ are distinct. (When $\alpha$ is the root, $\psi_{n}^{\alpha}$ is $\psi_{n}$.)

We call this rather heavy structure a nested configuration. We define charges 
and forces by

$$
\begin{gathered}
Q_{\alpha, k}=\sum_{i \in \alpha} Q_{i, k} \quad 1 \leq k \leq N \\
F_{\beta}^{\alpha}=\sum_{k=1}^{N} \sum_{\gamma \neq \beta} \frac{Q_{\beta, k} Q_{\gamma, k}}{\overline{p_{\beta}^{\alpha}}-\overline{p_{\gamma}^{\alpha}}}
\end{gathered}
$$

where $\beta$ and $\gamma$ are sons of $\alpha$.

The following theorem generalises Theorem 5 to the nested case.

Theorem 6 (nested case). In the above setting,

1) each node $\alpha$ is balanced, in the sense that for all sons $\beta$ of $\alpha, F_{\beta}^{\alpha}=0$.

2) For each node $\alpha$, there exists numbers $H_{k}^{\alpha}$ such that the following holds: Consider a leaf $i \in \alpha$ such that $M_{i, \infty}$ has ends at levels $k$ and $k+1$. Let $\alpha=\alpha_{0}, \alpha_{1}, \cdots, \alpha_{r}=i$ be the descending path from the node $\alpha$ to the leaf $i$.

Then there exists non-negative numbers $\rho_{1}, \cdots, \rho_{r}$ such that

$$
\sum_{s=1}^{r} \rho_{s}=1 \quad \text { and } \quad H_{k}^{\alpha}=\sum_{s=1}^{r} \rho_{s}\left(Q_{\alpha_{s}, k+1}-Q_{\alpha_{s}, k}\right) .
$$

In other words, point 2 means that $H_{k}^{\alpha}$ is in the smallest interval containing all the number $Q_{\alpha_{s}, k+1}-Q_{\alpha_{s}, k}$ that we encounter while descending the tree from $\alpha$ to $i$ ( $\alpha$ excluded). As was explained after Theorem 5 , this gives useful inequalities.

Proof. The first point is clear from the proof of Proposition 3. For the second point, we have by the proof of Proposition 4

$$
\begin{gathered}
\operatorname{Re} \int_{A_{k, n}^{\alpha_{s-1}}}^{A_{k, n}^{\alpha_{s}}} d h_{n} \sim Q_{\alpha_{s}, k} \log \frac{\lambda_{n}^{\alpha_{s-1}}}{\lambda_{n}^{\alpha_{s}}} \quad 1 \leq s \leq r-1 . \\
\operatorname{Re} \int_{A_{k, n}^{\alpha_{r-1}}}^{A_{k+1, n}^{\alpha_{r}-1}} d h_{n} \sim\left(Q_{\alpha_{r}, k}-Q_{\alpha_{r}, k+1}\right) \log \lambda_{n}^{\alpha_{r-1}} .
\end{gathered}
$$

Let us define $\lambda_{n}^{\alpha_{r}}=1$. This gives

$$
\frac{1}{\log \lambda_{n}^{\alpha_{0}}} \operatorname{Re} \int_{A_{k, n}^{\alpha_{0}}}^{A_{k+1, n}^{\alpha_{0}}} d h_{n} \sim \sum_{s=1}^{r}\left(Q_{\alpha_{s}, k}-Q_{\alpha_{s}, k+1}\right) \frac{\log \lambda_{n}^{\alpha_{s-1}}-\log \lambda_{n}^{\alpha_{s}}}{\log \lambda_{n}^{\alpha_{0}}} .
$$

Passing to a subsequence, we may assume that for each $1 \leq s \leq r$,

$$
\rho_{s}=\lim \frac{\log \lambda_{n}^{\alpha_{s-1}}-\log \lambda_{n}^{\alpha_{s}}}{\log \lambda_{n}^{\alpha_{0}}}
$$

exists. This gives the result. 


\section{Applications}

We say a configuration is trivial if it consists of one single point, which means that the sequence $M_{n}$ converges to one single surface $M_{1, \infty}$. The idea to prove compactness theorems is to classify all possible limit configurations, and use the hypotheses to rule out all non-trivial ones.

\subsection{The quadratic equation}

The following equation is the key to all our classification results. The basic idea is that it is quadratic and the charges are real, so in some cases it has no solutions.

Theorem 7. The charges satisfy

$$
\sum_{k=1}^{N} \sum_{i<j} Q_{i, k} Q_{j, k}=0
$$

Proof. First assume the configuration is non-singular. Then we write

$$
0=\sum_{i} \overline{p_{i}} F_{i}=\sum_{k} \sum_{i} \sum_{j \neq i} \overline{p_{i}} \frac{Q_{i, k} Q_{j, k}}{\overline{p_{i}}-\overline{p_{j}}}=\sum_{k} \sum_{i<j} Q_{i, k} Q_{j, k} .
$$

In the case of a singular configuration of depth 2 (namely when all sub-configurations are non-singular) we obtain, from $F_{i}^{\alpha}=0$ and $F_{\alpha}=0$ respectively

$$
\forall \alpha, \quad \sum_{k} \sum_{\substack{i, j \in \alpha \\ i<j}} Q_{i, k} Q_{j, k}=0 \quad \text { and } \quad \sum_{k} \sum_{\alpha<\beta} Q_{\alpha, k} Q_{\beta, k}=0 .
$$

Here the notation $\alpha<\beta$ simply means that we sum on all unordered pairs $\{\alpha, \beta\}$. Now

$$
\sum_{k} \sum_{i<j} Q_{i, k} Q_{j, k}=\sum_{k} \sum_{\alpha} \sum_{\substack{i, j \in \alpha \\ i<j}} Q_{i, k} Q_{j, k}+\sum_{k} \sum_{\alpha<\beta} \underbrace{\sum_{i \in \alpha} Q_{i, k}}_{Q_{\alpha, k}} \underbrace{\sum_{j \in \beta} Q_{j, k}}_{Q_{\beta, k}}=0 .
$$

Clearly the result follows in general by induction on the depth of the nested configuration.

\subsection{A criterion for uniform speed}

The levels of the ends of a limit surface $M_{i, \infty}$ form a set of consecutive integers, for if there were a gap at some level $k, M_{i, n}$ would cross the unbounded domain $\Omega_{k, n}$ which contradicts embeddedness. In this section we prove 
Proposition 5. Consider some level $k$ and assume that some limit surface $M_{i, \infty}$ has ends at levels $k$ and $k+1$ whose logarithmic growths are equal: $Q_{i, k}=Q_{i, k+1}$. Then $Q_{k+1} \leq Q_{k}$.

This implies as a corollary:

Theorem 8. Assume that the surfaces $M_{n}$ have uniformly separated ends. Then the sequence $\left(M_{n}\right)_{n}$ has uniform speed, and all the limit surfaces $M_{i, \infty}$ have separated ends.

Indeed, if the sequence $\left(M_{n}\right)_{n}$ does not have uniform speed, then at least one limit surface $M_{i, \infty}$ is flat so all its ends satisfies $Q_{i, k}=0$. If the ends are uniformly separated then $Q_{k}<Q_{k+1}$.

\subsubsection{Proof in the non-singular case}

First assume that the limit configuration is non-singular. We shall use the following doubtful but very convenient notation:

$$
\Delta Q_{i, k}=Q_{i, k+1}-Q_{i, k}
$$

I claim that $\forall j, \Delta Q_{j, k} \leq 0$. Indeed, if $M_{j, \infty}$ has ends at levels $k$ and $k+1$, then $\Delta Q_{j, k}=\Delta Q_{i, k}=0$ by the charge equation (Theorem 4). Else, either $M_{j, \infty}$ has its top end at level $k$, in which case $\Delta Q_{j, k}=-Q_{j, k} \leq 0$, or its bottom end at level $k+1$, in which case $\Delta Q_{j, k}=Q_{j, k+1} \leq 0$, or no end at level $k$ nor $k+1$, in which case $\Delta Q_{j, k}=0$. Summing on $j$ gives $\Delta Q_{k} \leq 0$.

\subsubsection{Proof of Proposition 5 in the singular case}

In the case of singular configuration, we argue by induction on the depth $r$ of the configuration. So assume that we have proven the proposition for all configurations of depth $<r$ and consider a configuration of depth $r$. Without loss of generality we may assume that all leaves of the tree have the same depth $r$, by introducing if necessary trivial sub-configurations (namely, configurations with only one point).

Let $\alpha_{0}$ be the root of the tree. Let $i$ be given in the hypothesis of the proposition, and let $\alpha_{0}, \alpha_{1}, \cdots, \alpha_{r}=i$ be the descending path from the root to $i$. By the induction hypothesis, we have $\Delta Q_{\alpha_{s}, k} \leq 0$ for each $s \geq 1$, because we may see the sub-configuration $\alpha_{s}$ as a configuration of depth $<r$. Hence

$$
H_{k}=\rho_{r} \Delta Q_{\alpha_{r}, k}+\cdots+\rho_{1} \Delta Q_{\alpha_{1}, k} \leq 0 .
$$

Consider now any leaf $j$ and let $\alpha_{0}, \beta_{1}, \beta_{2} \cdots, \beta_{r}=j$ be the descending path from the root to $j$. I claim that there exists non-negative numbers $\rho_{1}, \cdots, \rho_{r}$, not all zero, such that

$$
\rho_{r} \Delta Q_{\beta_{r}, k}+\rho_{r-1} \Delta Q_{\beta_{r-1}, k}+\cdots+\rho_{1} \Delta Q_{\beta_{1}, k} \leq 0 .
$$


If $j \in I(k) \cap I(k+1)$, this follows from $H_{k} \leq 0$ and Theorem 6. Else we have as above $\Delta Q_{j, k} \leq 0$, so the claim holds with $\rho_{r-1}=\cdots=\rho_{1}=0$.

From this we want to deduce that

$$
\rho_{r-1}^{\prime} \Delta Q_{\beta_{r-1}, k}+\cdots+\rho_{1}^{\prime} \Delta Q_{\beta_{1}, k} \leq 0
$$

for some other non-negative numbers $\rho_{1}^{\prime}, \cdots, \rho_{r-1}^{\prime}$, not all zero. We fix $\beta_{r-1}$ and consider all its sons $\beta_{r}$. (The numbers $\rho_{1}, \cdots, \rho_{r}$ depend on $\beta_{r}$.) If there exists a son $\beta_{r}$ of $\beta_{r-1}$ such that $\rho_{r}=0$, then we are done. Else we divide by $\rho_{r}$ to obtain

$$
\Delta Q_{\beta_{r}, k}+\frac{\rho_{r-1}}{\rho_{r}} \Delta Q_{\beta_{r-1}, k}+\cdots+\frac{\rho_{1}}{\rho_{r}} \Delta Q_{\beta_{1}, k} \leq 0 .
$$

Summing on all the sons $\beta_{r}$ of $\beta_{r-1}$ we obtain

$$
\Delta Q_{\beta_{r-1}, k}+\rho_{r-1}^{\prime \prime} \Delta Q_{\beta_{r-1}, k}+\cdots+\rho_{1}^{\prime \prime} \Delta Q_{\beta_{1}, k} \leq 0
$$

for some non-negative numbers $\rho_{r-1}^{\prime \prime}, \cdots, \rho_{1}^{\prime \prime}$. This proves (1). Now iterating this summation process we obtain by induction that $\Delta Q_{\beta_{1}, k} \leq 0$. So we have proven that for all sons $\beta$ of the root, $\Delta Q_{\beta, k} \leq 0$. Summing on $\beta$ we obtain $\Delta Q_{k} \leq 0$.

\subsection{Classification of configurations with 3 ends}

In this section we assume that each surface $M_{n}$ has $N=3$ ends. Then each limit surface $M_{i, \infty}$ is either a minimal surface with three ends, or a catenoid with ends at levels 1 and 2, or a catenoid with ends at levels 2 and 3. In the later two cases we call it a catenoid at level 1 and 2, respectively. The size of a catenoid is the logarithmic growth of its top end (this is equal to the radius of its waist circle).

Proposition 6 (classification). Assume that $N=3$ and the sequence $\left(M_{n}\right)_{n}$ has uniform speed. Then the possible weak limits $\left\{M_{1, \infty}, \cdots, M_{m, \infty}\right\}$ are, up to normalisation (namely: changing indices $i$, scaling charges $Q_{i, k}$, translating/scaling/ rotating the $p_{i}$, and putting all surfaces $M_{n}$ upside down)

1) $m=1$, and $M_{1, \infty}$ is a minimal surface with three ends.

2) $m=r+1$ where $r \geq 2 . M_{1, \infty}, \cdots, M_{r, \infty}$ are catenoids at level 1 with size 1. $M_{r+1, \infty}$ is a catenoid at level 2 with size $r-1$. The configuration is as follows: $p_{1}, \cdots, p_{r}$ are the $r^{\text {th }}$ roots of unity, $p_{r+1}=0$.

3) $m=4$, all $M_{i, \infty}$ are catenoids of size 1 , at level 1 if $i=1,2$ and 2 if $i=3,4$. The configuration is given by $p_{1}=1, p_{2}=-1, p_{3}=a$ and $p_{4}=1 / a$ where $a \in \mathbb{C} \backslash\{0,1,-1\}$ is a free parameter.

4) $m=4$ and the configuration is singular with two sub-configurations: a subconfiguration of three catenoids of size 1 given by point 2) above with $r=2$, and one single catenoid $M_{4, \infty}$ at level 2 with size $c \in(0,1]$. Namely, if we write $\alpha=\{1,2,3\}$ for the sub-configuration, then $M_{1, \infty}$ and $M_{2, \infty}$ are catenoids at level 1 with size $1, M_{3, \infty}$ is a catenoid at level 2 with size 1 , $p_{1}^{\alpha}=1, p_{2}^{\alpha}=-1$ and $p_{3}^{\alpha}=0$. 
Case 2 is the configuration that we obtain as a limit of the Costa Hoffman Meeks family of genus $r-1$. This configuration is non-degenerate, in the sense that it admits no non-trivial infinitesimal deformation (see [11]). As a consequence, if $\left(M_{n}\right)_{n}$ converges to this configuration, then $M_{n}$ must be a Costa Hoffman Meeks surface for $n$ large enough (see [11] - this follows from uniqueness in the implicit function theorem).

Case 3 is the possible limit of a family of minimal surfaces of genus 2 and 3 ends, known as Horgan surfaces, whose existence remains doubtful (see [11]). This configuration admits a non-trivial deformation so is degenerate. Case 4 may be seen as a limit case of case 3 when $a \rightarrow 0$. It is illustrated in figure 4 .

Before proving the proposition, let us prove Theorem 2 as a corollary. Let $\left(M_{n}\right)_{n}$ be a sequence of minimal surfaces as in this theorem. Since the ends are uniformly separated, the surface has uniform speed by Theorem 8 , so we are in one of the four cases of the above classification. Case 3 and 4 are excluded because $M_{n}$ would have genus 2 . Case 2 is also excluded because in this case, $M_{n}$ would be a Costa Hoffman Meeks surface of genus $r-1$ for $n$ large enough. Therefore, the only possibility is case 1 , which proves the theorem.

Remark 2. If we remove the hypothesis that the sequence has uniform speed, then many other weak limits are possible (or at least, we cannot rule them out). Here is an example: $m=2, M_{1, \infty}$ is a 3 -ended surface with $Q_{1,1}=Q_{1,2}<Q_{1,3}$ and $M_{2, \infty}$ is a catenoid at level 1 with size 0 . There might also be more catenoids of size 0 at level 1 , which gives examples of arbitrary genus $\geq 2$ (see figure 2 ). These examples do not have uniformly separated ends of course.

\subsubsection{Proof in the non-singular case}

We first prove the proposition in the non-singular case. Let $n_{1}, n_{2}$ and $n_{3}$ be respectively the number of catenoids at level 1 , at level 2 , and the number of minimal surfaces with three ends. For a catenoid of size $c$ with ends at levels $k$ and $k+1$ we have $Q_{i, k}=-c$ and $Q_{i, k+1}=c$ so $Q_{i, k+1}-Q_{i, k}=2 c$. By the charge equation (Theorem 4), all catenoids at the same level have the same size. Let $c_{1}>0$ and $c_{2}>0$ be the size of the catenoids at level 1 and 2 . Let $J$ be the set of indices $i$ such that $M_{i, \infty}$ has three ends. The quadratic equation (Theorem 7) gives an equation of the form $A+B+C=0$ where

$$
\begin{gathered}
A=\sum_{k=1}^{3} \sum_{\substack{i, j \in J \\
i<j}} Q_{i, k} Q_{j, k}, \\
B=\sum_{i \in J} n_{1} c_{1}\left(Q_{i, 2}-Q_{i, 1}\right)+n_{2} c_{2}\left(Q_{i, 3}-Q_{i, 2}\right), \\
C=n_{1}\left(n_{1}-1\right) c_{1}^{2}+n_{2}\left(n_{2}-1\right) c_{2}^{2}-n_{1} n_{2} c_{1} c_{2} .
\end{gathered}
$$

The idea is to obtain informations by proving each term is non-negative. 
SteP 1. We prove that $A \geq 0$ with equality only if $n_{3} \leq 1$. If $i \in J$ then $Q_{i, 1} \leq Q_{i, 2} \leq Q_{i, 3}$ and at least one inequality is strict. Moreover $Q_{i, 1}<0$ and $Q_{i, 3}>0$. This gives

$$
Q_{i, 1} Q_{j, 1}+Q_{i, 2} Q_{j, 2}+Q_{i, 3} Q_{j, 3} Q_{i, 2}\left(Q_{j, 1}+Q_{j, 2}+Q_{j, 3}\right)=0 .
$$

This proves the claim.

SteP 2. We classify the case $n_{3} \geq 1$. By the charge equation (Theorem 4 ), we have if $i \in J, Q_{i, 2}-Q_{i, 1}=2 c_{1}$ and $Q_{i, 3}-Q_{i, 2}=2 c_{2}$ so all terms in $B$ are non-negative. Keeping only one term we get

$$
B \geq 2 n_{1} c_{1}^{2}+2 n_{2} c_{2}^{2} .
$$

This gives

$$
B+C \geq \frac{1}{2}\left[\left(n_{1} c_{1}-n_{2} c_{2}\right)^{2}+n_{1}\left(n_{1}+2\right) c_{1}^{2}+n_{2}\left(n_{2}+2\right) c_{2}^{2}\right] .
$$

All terms are non-negative so all must be zero, hence $n_{1}=n_{2}=0$ and $A=0$ which gives $n_{3}=1$. This is case 1 of the proposition.

SteP 3 . We classify the case $n_{3}=0$. Only the $C$ term remains which we rewrite as

$$
C=\frac{1}{2}\left[\left(n_{1} c_{1}-n_{2} c_{2}\right)^{2}+n_{1}\left(n_{1}-2\right) c_{1}^{2}+n_{2}\left(n_{2}-2\right) c_{2}^{2}\right]=0 .
$$

If $n_{1} \geq 2$ and $n_{2} \geq 2$ then all terms are non-negative so $n_{1}=n_{2}=2$ and $c_{1}=c_{2}$. This is case 3 of the proposition. If $n_{1}=1$ then $c_{1}=\left(n_{2}-1\right) c_{2}$ so $c_{1}>0$ implies $n_{2} \geq 2$. This is case 2 upside down. The case $n_{2}=1$ is similar. It remains to classify the possible configurations $p_{1}, \cdots, p_{m}$ in each case. This is done in [11].

\subsubsection{Proof of Proposition 6 in the singular case}

Assume the configuration is singular. First observe that there are no non-trivial configurations with $N=2$ - this clearly follows from the quadratic equation. Hence any non-trivial sub-configuration must have 3 ends. As far as forces are concerned, each sub-configuration may be seen as one single surface with three ends of logarithmic growths $Q_{\alpha, 1}, Q_{\alpha, 2}$ and $Q_{\alpha, 3}$. So in this section, we see each sub-configuration as a "fake" 3 -ended surface. The difference between a subconfiguration and a "true" 3 -ended surface is that for a sub-configuration, the charge equation $H_{k}=Q_{i, k+1}-Q_{i, k}$ does not hold and must be replaced by some inequalities as explained after Theorem 5 .

As in the previous section, let $n_{1}$ and $n_{2}$ be the number of catenoids at level 1 and 2 which correspond to non-singular points of the configuration. Let $n_{3}$ be the number of 3 -ended surfaces plus the number of sub-configurations (or "fake" 3 -ended surfaces).

The equation $A+B+C=0$ still holds provided we replace $J$ by $J \cup J^{\prime}$ where $J^{\prime}$ is the set of $\alpha$ corresponding to sub-configurations. If $\alpha$ is a non-trivial 
sub-configuration, then it must match one of the cases 2,3,4 of Proposition 6 . Observe that in all cases, the logarithmic growths of the sub-configuration satisfy $Q_{\alpha, 1} \leq Q_{\alpha, 2} \leq Q_{\alpha, 3}$ with at least one strict inequality. Hence, we conclude as in step 1 that $A \geq 0$, with equality if $n_{3} \leq 1$.

To estimate the $B$ term in step 2 , we need to obtain some inequalities for $Q_{\alpha, k+1}-Q_{\alpha, k}$. We deal with each case of the proposition separately.

CAse 2. Assume the configuration contains a sub-configuration $\alpha$ given by case 2 of the classification. If $i \in \alpha$ is a catenoid at level 1 , we have by point $2 \mathrm{~b}$ ) of Theorem 5

$$
Q_{i, 2}-Q_{i, 1}=2, \quad Q_{\alpha, 2}-Q_{\alpha, 1}=r+1 \quad \Longrightarrow \quad 2 \leq 2 c_{1} \leq r+1 .
$$

If $j \in \alpha$ is the catenoid at level 2 , we have

$$
Q_{j, 3}-Q_{j, 2}=2 r-2, \quad Q_{\alpha, 3}-Q_{\alpha, 2}=r-2 \quad \Longrightarrow r-2 \leq 2 c_{2} \leq 2 r-2 .
$$

These inequalities are enough to conclude. Indeed, we obtain

$$
Q_{\alpha, 2}-Q_{\alpha, 1} \geq 2 c_{1}, \quad Q_{\alpha, 3}-Q_{\alpha, 2} \geq \frac{r-2}{r-1} c_{2} .
$$

We use this to estimate $B$, this gives

$$
B+C \geq \frac{1}{2}\left[\left(n_{1} c_{1}-n_{2} c_{2}\right)^{2}+c_{1}^{2}\left(n_{1}^{2}+2 n_{1}\right)+c_{2}^{2} n_{2}\left(n_{2}-\frac{2}{r-1}\right)\right] .
$$

If $n_{2}=0$, or $n_{2}=1$ and $r \geq 3$, or $n_{2} \geq 2$, the last term is non-negative, so all must be zero, which gives $n_{1}=n_{2}=0$. The only remaining case is $n_{2}=1$ and $r=2$. In this case, we obtain, from the first inequalities, that $c_{2} \leq c_{1}$. This gives

$$
B+C \geq \frac{1}{2}\left[\left(n_{1} c_{1}-c_{2}\right)^{2}+c_{1}^{2}\left(n_{1}^{2}+2 n_{1}\right)-c_{1}^{2}\right] .
$$

If $n_{1} \geq 1$ this is positive, so $n_{1}=0$. Hence $A=0$ so $n_{3}=1$. This is case 4 of the classification.

CAsE 3. Assume the configuration contains a sub-configuration $\alpha$ given by case 3 of the classification. If $i \in \alpha$ is a catenoid at level $k \in\{1,2\}$, we have

$$
Q_{i, k+1}-Q_{i, k}=Q_{\alpha, k+1}-Q_{\alpha, k}=2, \quad \Longrightarrow 2 c_{k}=2 .
$$

Hence in this case, the sub-configuration behaves as a "true" 3 -ended surface. As in the non-singular case, we conclude that this case cannot happen.

CAsE 4. Assume the configuration contains a sub-configuration $\beta$ given by case 4 the classification, so that we have nested configurations $\alpha \subset \beta$. If $i \in \alpha$ is a catenoid at level 1 , we have by Theorem 6

$$
Q_{i, 2}-Q_{i, 1}=2, \quad Q_{\alpha, 2}-Q_{\alpha, 1}=3, \quad Q_{\beta, 2}-Q_{\beta, 1}=3-c \quad \Longrightarrow 2 \leq 2 c_{1} \leq 3 .
$$

If $i \in \alpha$ is the catenoid at level 2, we have

$$
Q_{i, 3}-Q_{i, 2}=2, \quad Q_{\alpha, 3}-Q_{\alpha, 2}=0, \quad Q_{\beta, 3}-Q_{\beta, 2}=2 c \Longrightarrow 0 \leq 2 c_{2} \leq 2 .
$$


Hence $Q_{\beta, 2}-Q_{\beta, 1} \geq 4 c_{1} / 3$ and $c_{2} \leq c_{1}$. We conclude as in case 2 that $n_{2}=1$ and $n_{1}=0$. Then since $Q_{\beta, 3}-Q_{\beta, 2}>0$, we have $B+C>0$, so this case cannot happen.

Remark 3. From embeddedness, we have $Q_{k} \leq Q_{k+1}$. We were careful not to use this information in the proof. Indeed, it is a priori not true that for a subconfiguration, one has $Q_{\alpha, k} \leq Q_{\alpha, k+1}$. We conclude a posteriori, from the classification, that this is true.

\subsection{Classification in the low genus case}

In this section we prove Theorem 1. We fix some genus $G$ and assume that conjecture 1 holds for all genus $G^{\prime}<G$, namely, any embedded minimal surface of genus $G^{\prime}$ has at most $G^{\prime}+2$ ends. We consider a sequence $\left(M_{n}\right)_{n}$ of embedded minimal surfaces of genus $G$ and with $N$ ends which are counterexamples to the conjecture, namely $N \geq G+3$. We want to prove that the limit configuration is trivial (only one surface) so $\left(M_{n}\right)_{n}$ converges to a counterexample. So we assume the limit configuration is non-trivial and we obtain a contradiction. Claims 1 and 2 below reduce the problem to the analysis of the configurations of type 1-2-2 $\cdots 1$ (by which we mean that there is one catenoid at level 1, two catenoids at level 2, and so on). We can then easily rule out these configurations by proving that the quadratic equation is negative.

It is interesting to compare this with the argument of A. Ros in [8]. By a completely different argument he could rule out configurations of type 1-1 (which is a particular case of the above case with no 2's). So he obtained similar, but weaker, compactness results.

Claim 1. There exists levels $a$ and $b$, with $a \leq b$, such that the following holds:

- there is precisely one surface $M_{i, \infty}$ between levels $a-1$ and $a$ (by this we mean that $M_{i, \infty}$ is the only surface which has ends at levels $a-1$ and $a$, it may have more ends).

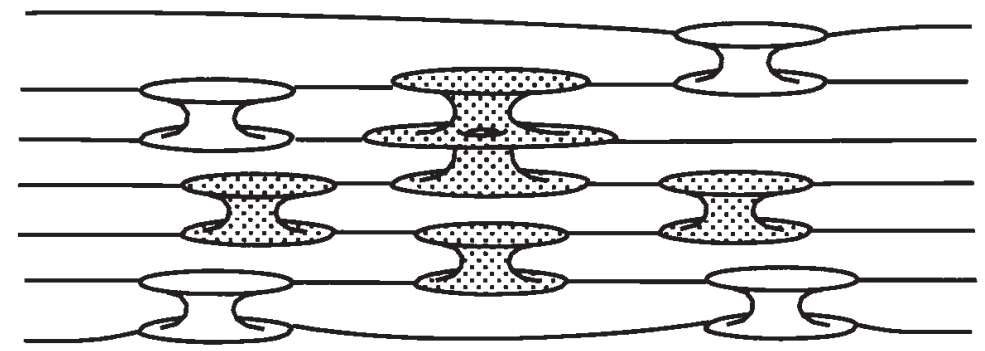

FIG. 7. A sequence of minimal surfaces of genus 4 with 7 ends. We keep only the shaded surfaces in Claim 2. 
- For each $k$ such that $a \leq k<b$, there are precisely two surfaces between levels $k$ and $k+1$, and these are catenoids.

- There is one surface between levels $b$ and $b+1$, which we call $M_{j, \infty}$, with $j \neq i$. It follows that $M_{i, \infty}$ has its top end at level $a$ and $M_{j, \infty}$ has its bottom end at level $b$. Both $M_{i, \infty}$ and $M_{j, \infty}$ may, or may not, be catenoids.

Claim 2. Remove all surfaces which are below $a$, and all surfaces which are above $b+1$ (namely, keep only $M_{i, \infty}, M_{j, \infty}$ and the middle catenoids, if any). Then this new configuration is still balanced.

Claim 3. The configuration of Claim 2 cannot be balanced.

Proof of Claim 1. Let $G_{i}$ and $N_{i}$ be the genus and number of ends of $M_{i, \infty}$. The genus of $M_{n}$ is given by

$$
G=\sum_{i}\left(G_{i}+N_{i}-1\right)-N+1
$$

For $1 \leq k \leq N-1$, define

$a_{i, k}=\left\{\begin{array}{l}1 \text { if } M_{i, \infty} \text { has its top end at level } k+1, \\ 2 \text { if } M_{i, \infty} \text { has ends at levels } k \text { and } k+1, \text { and } k+1 \text { is not the top end, } \\ 0 \text { else. }\end{array}\right.$

Then

$$
\begin{gathered}
\sum_{k=1}^{N-1} a_{i, k}=2 N_{i}-3 \\
a_{k}=\sum_{i} a_{i, k} \geq 1 \quad \text { because } M_{n} \text { is connected. }
\end{gathered}
$$

If there exists $i$ such that $G_{i}=G$, then all other surfaces must have genus zero so be catenoids, and there is only one per level, so the claim holds with $a=b$ (because the configuration is non-trivial).

Else we have $G_{i}<G$ for all $i$, so $G_{i} \geq N_{i}-2$. This gives

$$
2 N-4 \geq G+N-1=\sum_{i}\left(G_{i}+N_{i}-1\right) \geq \sum_{i}\left(2 N_{i}-3\right)=\sum_{i} \sum_{k} a_{i, k}=\sum_{k} a_{k} .
$$

We have $N-1$ integers $a_{k} \geq 1$ to make a total $\leq 2(N-1)-2$, so the sequence $a_{1}, \cdots, a_{N-1}$ must contains a subsequence of the form $1,2, \cdots, 2,1$ (the number of 2 may be zero). The claim easily follows.

Proof of Claim 2. The claim follows from the following

Lemma 3 (pruning the configuration). Assume we have a balanced configuration such that for some level $a$, there is only one surface $M_{i, \infty}$ between levels a and $a+1$. Let $I^{-}$(respectively $I^{+}$) be the set of $j$ such that $M_{j, \infty}$ has all its ends at level $\leq a$ (respectively $\geq a+1)$. Then the configuration obtained by removing all 
surfaces $M_{j}, j \in I^{-}$is still balanced. In the same way, the configuration obtained by removing all surfaces $M_{j}, j \in I^{+}$, is balanced.

Proof. First assume the configuration is non-singular. Let

$$
F_{i}^{ \pm}=\sum_{k=1}^{N} \sum_{j \in I^{ \pm}} \frac{Q_{i, k} Q_{j, k}}{\overline{p_{i}}-\overline{p_{j}}}
$$

so that $F_{i}=F_{i}^{+}+F_{i}^{-}$. Then

$$
F_{i}^{-}+\sum_{j \in I^{-}} F_{j}=\sum_{k=1}^{N-1} \sum_{\substack{j, \ell \in I^{-} \cup\{i\} \\ j \neq \ell}} \frac{Q_{j, k} Q_{\ell, k}}{\overline{p_{j}}-\overline{p_{\ell}}}=0
$$

because the last sum is zero whatever the value of the $p_{j}$. Hence $F_{i}^{-}=F_{i}^{+}=0$. When we remove all surfaces below level $a$, namely $M_{j, \infty}$ with $j \in I^{-}$, the forces $F_{j}$ for $j \in I^{+}$do not change (because the surfaces below level $a$ do not interact with the surfaces above level $a+1)$ and $F_{i}$ is replaced by $F_{i}^{+}$. Hence the configuration is still balanced.

In the singular case, we need to prove that the configuration is balanced in the sense of Theorem 6 . Let $\alpha$ be the class of $i$. Let $I^{-}$(respectively $I^{+}$) be the set of classes $\beta$ such that all surfaces $M_{j, \infty}$ for $j \in \beta$ have ends below level $a$ (respectively above $a+1$ ). Then as above, we have $F_{\alpha}^{+}=0$. Observe that pruning only changes the charges $Q_{\alpha, k}$ for $k \leq a$, but these do not interact with the points $p_{\beta}$ for $\beta \in I^{+}$. Hence, the configuration remains balanced after pruning, in the sense $F_{\beta}=0$. It is clear that each sub-configuration remains balanced, simply forget about the rest of the configuration and use the same argument.

Proof of Claim 3. For $a \leq k<b$, let $c_{k}$ and $c_{k}^{\prime}$ be the sizes of the two catenoids at level $k$. Note that we do not assume that the configuration is non-singular here, so we cannot say that $c_{k}=c_{k}^{\prime}$. We do not assume either that it has uniform speed, so we might have $c_{k}=c_{k}^{\prime}=0$. The quadratic equation gives

$$
-Q_{i, a}\left(c_{a}+c_{a}^{\prime}\right)+Q_{j, b}\left(c_{b-1}+c_{b-1}^{\prime}\right)+\sum_{k=a}^{b-1} 2 c_{k} c_{k}^{\prime}-\sum_{k=a}^{b-2}\left(c_{k}+c_{k}^{\prime}\right)\left(c_{k+1}+c_{k+1}^{\prime}\right)=0 .
$$

We rewrite this as

$$
\begin{aligned}
\left(2 c_{a} c_{a}^{\prime}-Q_{i, a}\left(c_{a}+c_{a}^{\prime}\right)\right) & +Q_{j, b}\left(c_{b-1}+c_{b-1}^{\prime}\right) \\
& +\sum_{k=a}^{b-2}\left(2 c_{k+1} c_{k+1}^{\prime}-\left(c_{k+1}+c_{k+1}^{\prime}\right)\left(c_{k}+c_{k}^{\prime}\right)\right)=0 .
\end{aligned}
$$

We want to prove that each term is non-positive. Since $M_{n}$ is embedded we have $Q_{k} \leq Q_{k+1}$ for all $1 \leq k \leq N-1$ (here $Q_{k}$ is the logarithmic growth before the 
pruning operation). We have

$$
\begin{gathered}
Q_{a-1} \geq Q_{i, a-1} \geq-Q_{i, a} \\
Q_{a}=Q_{i, a}-c_{a}-c_{a}^{\prime} \\
Q_{k}=c_{k-1}+c_{k-1}^{\prime}-c_{k}-c_{k}^{\prime} \quad \text { for } a+1 \leq k \leq b-1
\end{gathered}
$$

This gives for $a \leq k \leq b-2$,

$$
\begin{gathered}
-c_{k}-c_{k}^{\prime} \leq Q_{k} \leq Q_{k+1}=c_{k}+c_{k}^{\prime}-c_{k+1}-c_{k+1}^{\prime} \Longrightarrow-2\left(c_{k}+c_{k}^{\prime}\right) \leq-\left(c_{k+1}+c_{k+1}^{\prime}\right) \\
\Longrightarrow 2 c_{k+1} c_{k+1}^{\prime}-\left(c_{k+1}+c_{k+1}^{\prime}\right)\left(c_{k}+c_{k}^{\prime}\right) \leq-\frac{1}{2}\left(c_{k+1}-c_{k+1}^{\prime}\right)^{2} \leq 0 .
\end{gathered}
$$

In the same way,

$$
-Q_{i, a} \leq Q_{a-1} \leq Q_{a} \leq Q_{i, a}-c_{a}-c_{a}^{\prime}
$$

implies that

$$
2 c_{a} c_{a}^{\prime}-Q_{i, a}\left(c_{a}+c_{a}^{\prime}\right) \leq 0 .
$$

Since $Q_{j, b} \leq 0$, all terms in (2) are non-positive, so all are zero. In particular, we have either $Q_{j, b}=0$, so $M_{j, b}$ is flat, or $c_{b-1}=c_{b-1}^{\prime}=0$, so the catenoids at level $b-1$ are flat. The following lemma shows that this cannot happen

Lemma 4. For each $k, 1 \leq k \leq N-1$, there is at least one surface $M_{i, \infty}$ which has ends at levels $k$ and $k+1$ and is not flat.

Proof. Assume to the contrary that there is some $k$ such that all surfaces which have ends at levels $k$ and $k+1$ are flat. Then $Q_{k} \geq 0$, with equality only if all surfaces which have their top end at level $k$ are flat. In the same way $Q_{k+1} \leq 0$, with equality only if all surfaces which have their bottom end at level $k+1$ are flat. Since $Q_{k} \leq Q_{k+1}$, this proves that all surfaces which have ends at levels $k-1$ and $k$, or $k+1$ and $k+2$, are flat. By induction we find that all surfaces are flat, which is impossible.

\section{References}

[1] C. Costa, Uniqueness of minimal surfaces embedded in $\mathbb{R}^{3}$ with total curvature $12 \pi$, Journal Diff. Geom. 30 (3) (1989), 597-618.

[2] D. Hoffman and H. Karcher, Complete Embedded Minimal Surfaces of Finite Total Curvature, in: R. Osserman (ed.), Geometry V, 5-93, Encyclopedia of Math. Sci. 90, SpringerVerlag, 1997.

[3] Lehto, Virtanen, Quasiconformal mappings in the plane, second edition, Springer-Verlag, 1973.

[4] F. Lopez and A. Ros, On embedded complete minimal surfaces of genus zero, Journal Diff. Geom. 33 (1991), 293-300.

[5] W. H. Meeks, J. Perez and A. Ros, Uniqueness of the Riemann minimal example, Invent. Math. 133, no 1 (1998), 107-132. 
[6] J. Perez and A. Ros, Properly embedded minimal surfaces with finite total curvature, Preprint, 2000.

[7] J. Perez and A. Ros, The space of properly embedded minimal surfaces with finite total curvature, Indiana Univ. Math. J. 45 (1996), 177-204.

[8] A. Ros, Compactness of spaces of properly embedded minimal surfaces with finite total curvature, Indiana Univ. Math. Journal $44 \mathrm{n}^{\mathrm{O}} 1$ (1995), 139-152.

[9] R. Sibner, Remarks on the Koebe Kreisnormierungsproblem, Comm. Math. Helv. 43 (1968), 289-295.

[10] R. Schoen, Uniqueness, symmetry and embeddedness of minimal surfaces, Journal Diff. Geom. 18 (1983), 791-809.

[11] M. Traizet, An embedded minimal surface with no symmetries, Journal Diff. Geom. 60 (1) (2002), 103-153.

Martin Traizet

Département de Mathématiques

Université de Tours

37200 Tours

France

e-mail: martin@gargan.math.univ-tours.fr

(Received: May 27, 2003)

(10) To access this journal online:

(20) http://www.birkhauser.ch 